\title{
COMBINATORIAL CHARACTERIZATION OF SUPERCOMPACT CARDINALS
}

\author{
M. MAGIDOR
}

\begin{abstract}
It is proved that supercompact cardinals can be characterized by combinatorial properties which are generalizations of ineffability.
\end{abstract}

0. Introduction. $A \triangle B$ is the symmetric difference of $A$ and $B$. Greek letters will denote ordinals. $P_{k}(A)$ is the set of all nonempty subsets of $A$ of cardinality less than $k$. The cardinality of $A$ is $|A| .[A]^{\alpha}$ is the set of all subsets of $A$ of cardinality $\alpha$. An ultrafilter $U$ on $P_{k}(A)$ is normal if

(a) $U$ is $k$ complete;

(b) for every $a \in A\left\{P \mid P \in P_{k}(A), a \in P\right\} \in U$;

(c) any choice function on $P_{k}(A)$ is almost everywhere constant.

The definition of supercompact cardinal is due to Solovay [6]. A cardinal $k$ is $A$ supercompact if there is a normal ultrafilter on $P_{k}(A) \cdot k$ is supercompact if it is $A$ supercompact for all $A . R(\alpha)$ is the set of all sets of rank $<\alpha$. The purpose of this paper is to show that being a supercompact cardinal can be characterized by partition properties which are natural generalizations of those defining ineffable cardinal (JensenKunen [3]). By this we partially settle problem 4 of T. J. Jech [2]. We assume the reader knows the definition of closed subset of an ordinal, unbounded subset of an ordinal, and stationary set, as well as the basic properties of such sets (cf. Fodor [1]).

A cardinal $k$ is ineffable iff for any sequence $\left\{A_{\alpha}\right\}_{\alpha<k}$, such that $A_{\alpha} \subseteq \alpha$ for all $\alpha<k$, there is $A \subseteq k$ and $\left\{\alpha \mid \alpha<k, A \cap \alpha=A_{\alpha}\right\}$ is stationary in $k$.

An equivalent definition is the following partition property:

For any function $f:[k]^{2} \rightarrow 2$ there is a homogeneous stationary set for $f$ (that is, a stationary set $A$ such that $\left|f^{\prime \prime}[A]^{2}\right|=1$ ). An ineffable cardinal is weakly compact and therefore inaccessible.

1. Basic facts. A natural generalization of closed, unbounded and stationary sets are the following:

A subset $B$ of $P_{k}(A)$ is unbounded if for any $Q \in P_{k}(A)$. there is $P \in B$

Received by the editors March 22, 1972.

AMS (MOS) subject classifications (1970). Primary 02K 35 .

${ }^{1}$ This is a part of the author's Ph.D. thesis done under the supervision of Professor A. Lévy to whom the author is greatly indebted for his help and encouragement.

(c) American Mathematical Society 1974 
such that $Q \subseteq P$. A subset $B$ of $P_{k}(A)$ is closed if it is closed under unions of increasing chains such that their union is of cardinality less than $k$.

Note. $\quad k$ as a subset of $P_{k}(k)$ is a closed unbounded subset of $P_{k}(k)$ and a subset $B$ of $P_{k}(k)$ is stationary iff $B \cap k$ is a stationary subset of $k$ in the usual sense. (A subset of $P_{k}(A)$ is stationary if it intersects any closed unbounded subset of $P_{k}(A)$.)

This definition of closed set is a little different from Definition 3.1 in Jech [2], but the following lemma states that both definitions are equivalent.

Jech's definition is: A subset of $P_{k}(A)$ is closed if it is closed under unions of directed subsets whose union is of cardinality less than $k$. (A directed set $C$ is a set such that for every $P, Q$ in $C$ there is $T \in C$ and $P \cup Q \subseteq T$.) It is trivial that a set which is closed under union of directed subsets is closed. The other direction follows from:

Lemma (Solovay). A closed subset of $P_{k}(A)$ is closed under unions of directed subsets whose union is of cardinality less than $k$.

Proof. Let $B$ be a closed subset of $P_{k}(A)$ and let $C$ be a directed subset of $B$ such that $|\bigcup C|<k$. We want to prove $\bigcup C \in B$. We are going to prove it by induction on $|C|$.

(a) If $|C| \leqq \aleph_{0}$. let $P_{0}, P_{1}, \cdots, P_{n}, \cdots, n<w$, be an enumeration (possibly with repetitions) of the members of $C$. Define an increasing sequence of members of $C,\left\{P_{n}^{\prime}\right\}_{n<w}, P_{0}^{\prime}=P_{0}$. $P_{n+1}^{\prime}=$ a member of $C$ which includes $P_{n}^{\prime} \cup P_{n+1}$. $\bigcup P_{n}^{\prime} \in B$ ( $B$ is a closed set!). Therefore, $\cup P_{n} \in B$ because $\cup P_{n}^{\prime}=\bigcup P_{n}$.

(b) Suppose the lemma is true for any directed subsets of $B$ of cardinality $\left\langle|C|\right.$ and $|C|>\boldsymbol{\aleph}_{0}$. We construct by induction an increasing sequence of directed subsets of $B,\left\{C_{\alpha}\right\}_{\alpha<|C|}, C_{\alpha} \subseteq C$ and $\left|C_{\alpha}\right| \leqq \alpha+\aleph_{0}$. We use the fact that if $D \subseteq C$ then there is $D \subseteq D^{\prime} \subseteq C$ such that $D^{\prime}$ is directed and $\left|D^{\prime}\right| \leqq$ $|D|+\aleph_{0}$.

(The proof is by picking, for each two elements of $D$, an element of $C$ which includes their union, then iterating this process $\boldsymbol{\kappa}_{0}$ times and taking the union.)

Let $P_{0}, \cdots, P_{\alpha}, \cdots, \alpha<|C|$, be a well ordering of the elements of $C$. Define $C_{\alpha}: C_{0}=\left\{P_{0}\right\} ; C_{\alpha}=$ a directed subset of $C$ which includes the union of $\left\{C_{\beta}\right\}_{\beta<\alpha}$ and $P_{\alpha}$ and is of cardinality $\leqq\left|\bigcup_{\beta<\alpha} C_{\beta}\right|+\boldsymbol{K}_{0}$. (By induction $\left.\left|C_{\alpha}\right| \leqq \alpha+\aleph_{0}.\right)\left|C_{\alpha}\right|<|C|$ and $C_{\alpha}$ is a directed subset of $B$, by the induction hypothesis $P_{\alpha}^{\prime}=\bigcup C_{\alpha} \in B$. By $\left\{P_{\alpha}^{\prime}\right\}_{\alpha<|C|}$ is an increasing chain of elements of $B$, which means $\bigcup\left\{P_{\alpha}^{\prime}\right\}_{\alpha<|C|} \in B$ but $\bigcup\left\{P_{\alpha}^{\prime}\right\}_{\alpha<|C|}=\bigcup C$. Q.E.D.

Theorem 3.2 of Jech [2] is (for $k$ regular):

THEOREM. (a) The sets $P=\left\{Q \in P_{k}(\lambda): Q \supseteq P\right\}$ are closed unbounded. 
(b) The intersection of less than $k$ closed unbounded sets is closed unbounded.

(c) The diagonal intersection of closed unbounded sets is closed unbounded. (The diagonal intersection of $\left\{B_{a}\right\}_{a \in A}$ is $\left\{P \mid P \in P_{k}(A), P \in \bigcap_{a \in P} B_{a}\right\}$.)

(d) If $S$ is a stationary subset of $P_{k}(A)$ and $f$ is a choice function on $S$, then $f$ is constant on some stationary subset of $S$.

Theorem 3.3 of Jech [2] actually states that any member of a normal ultrafilter on $P_{k}(A)$ is stationary.

Definition. $k$ is $A$ ineffable if, for any function $f: P_{k}(A) \rightarrow P_{k}(A)$ such that $f(P) \subseteq P$ for all $P \in P_{k}(A)$, there is a subset of $A, B$, such that $\{P \mid f(P)=B \cap P\}$ is stationary.

(It is trivial that $k$ is $A$ ineffable if and only if it is $|A|$ ineffable.)

LEMMA. If $k$ is $A$ supercompact, then $k$ is $A$ ineffable.

Proof. Let $f$ be a function $f: P_{k}(A) \rightarrow P_{k}(A)$ such that $f(P) \subseteq P$ for all $P \in P_{k}(A)$, and let $U$ be a normal ultrafilter on $P_{k}(A)$. Define

$$
B=\{a \mid a \in A,\{P \mid a \in f(P)\} \in U\} .
$$

Claim. $\{P \mid B \cap P=f(P)\} \in U$. Suppose not, then define a choice function $g$ on $\{P \mid B \cap P \neq f(P)\} ; g(P)=$ the first member of $(B \cap P) \triangle f(P)$. Since $U$ is a normal ultrafilter, $g$ is almost everywhere constant $(\{\boldsymbol{P} \mid \boldsymbol{B} \cap P \neq f(\boldsymbol{P})\} \in U$ by assumption). Let $\boldsymbol{a}$ be the constant value of $\boldsymbol{g}$. then either

$$
\{P \mid a \in B \cap P-f(P)\} \in U \text { or }\{P \mid a \in f(P)-B \cap P\} \in U .
$$

In the first case, $a \in B$, but it contradicts $\{P \mid a \in f(P)\} \notin U$. In the second case $a \notin B$ but it contradicts the definition of $B$ and $\{P \mid a \in f(P)\} \in U$. Therefore, $\{P \mid B \cap P=f(P)\} \in U$, but any member of $U$ is a stationary subset of $P_{k}(A)$. Q.E.D.

In order to use Theorem 3.2 of Jech [2] we need that $k$ is regular, and that $k$ is $k$ ineffable iff $k$ is ineffable in the sense of Jensen and Kunen. Therefore, in order to have $k$ regular, it is enough that it is $k$ ineffable. When needed, we shall derive it from:

Lemma. If $k$ is $A$ ineffable and $B \subseteq A(B \neq \varnothing)$ then $k$ is $B$ ineffable.

Proof. Let $f$ be a function $f: P_{k}(B) \rightarrow P_{k}(B)$ such that for all $P \in P_{k}(B)$, $f(P) \subseteq P$. Let us define a function $g: P_{k}(A) \rightarrow P_{k}(A)$ by:

$$
\begin{array}{ll}
g(Q)=f(Q \cap B), & \text { if } Q \cap B \neq \varnothing, \\
g(Q)=\ell, & \text { if } Q \cap B=\varnothing .
\end{array}
$$


It is clear that $g(Q) \subseteq Q \cap B \subseteq Q$ for any $Q \in P_{k}(A)$. By assumption there is $C \subseteq A$ s.t. $\left\{Q \mid Q \in P_{k}(A), Q \cap C=g(Q)\right\}$ is stationary. $C \subseteq B$, otherwise pick $a \in C-B$ and some $Q$ such that $Q \cap C=g(Q)$ and $a \in Q$ which contradicts $g(Q) \subseteq Q \cap B$.

Claim. $\left\{P \mid P \in P_{k}(B), P \cap C=f(P)\right\}$ is stationary in $P_{k}(B)$. Otherwise there is a closed unbounded subset of $P_{k}(B), E$, such that for $P \in E$ $f(P) \neq P \cap C$.

Define $E^{\prime}=\left\{Q \mid Q \in P_{k}(A), Q \cap B \in E\right\} . E^{\prime}$ is closed unbounded in $P_{k}(A)$; therefore, there is $Q \in E^{\prime}$ such that $g(Q)=Q \cap C=f(Q \cap B)$. But $Q \cap B \in E$ and $Q \cap C=f(Q \cap B)$, hence a contradiction. Q.E.D.

\section{The main theorem.}

THEOREM. $k$ is a supercompact cardinal iff it is $A$ ineffable for all $A$.

ProOF. We proved in $\S 1$ that if $k$ is supercompact, it is $A$ ineffable for all $A$. For the other direction, we use the following definition:

Definimion. $k$ is $\beta$ reducible if $k<\beta$ and there is an $\alpha<\beta$ and an elementary embedding $j$ of $\langle R(\alpha), \epsilon\rangle$ into $\langle R(\beta), \epsilon\rangle$ such that $j$ is not the identity and such that if $\gamma$ is the first ordinal for which $j(\gamma)>\gamma$, then $j(\gamma)=k$. From Lemma 2 and Lemma 3 in Magidor [4] follows the following characterization of supercompact cardinal:

THEOREM 1. $k$ is supercompact iff it is $\beta$ reducible for all $k<\beta$.

Theorem 1 will follow from the next claim:

Claim. If $k<\beta$ and $k$ is $R(\beta)$ ineffable, then $k$ is $\beta$ reducible.

Proof of THE Claim.

LEMMA. Let $\langle A, E\rangle$ be a structure such that $E$ is well founded, and $\langle A, E\rangle$ is the elementary equivalent of $\langle R(\beta), E\rangle$ for some $\beta$, then $\langle A, E\rangle$ is isomorphic to a structure of the form $\langle R(\alpha), \epsilon\rangle$ iff for every $x \in A$ and $X \subseteq$ $\{y \mid y E x\}$, there is $t \in A$ such that $z E t$ iff $z \in X$. (See the proof of Theorem 2 in Magidor [4].)

Proof. If $\langle A, E\rangle$ is isomorphic to $\langle R(\alpha), \epsilon\rangle$ then the conclusion is trivial. For the other direction we use the fact that $\langle A, E\rangle$ is isomorphic to a structure of the form $\langle M, \epsilon\rangle$ where $M$ is transitive. $\langle M, \epsilon\rangle$ is elementary equivalent to $\langle R(\beta), \epsilon\rangle$ and therefore for every $\gamma \in M, R(\gamma) \cap M \in M$ (because $R(\gamma)$ is the set of sets of rank $<\gamma$ and the rank function is defined in $M$ and absolute). But by induction, we can prove that $R(\gamma) \cap M=R(\gamma)$ for $\gamma \in M$ and $M=\bigcup_{\gamma \in M} R(\gamma)$. Therefore, there is an $\alpha$ such that $M=$ $R(\alpha)$. Q.E.D. (Lemma)

Let $k$ be $R(\beta)$ ineffable. Let $B=\left\{P \mid P \in P_{k}(R(\beta)),\left\langle P, \in \cap P^{2}\right\rangle\right.$ is an elementary substructure of $\langle R(\beta), \epsilon\rangle\}$. Trivially $B$ is a closed unbounded subset of $P_{k}(R(\beta))$. 
Let $C=\left\{P \mid P \in B,\left\langle P, \epsilon \cap P^{2}\right\rangle\right.$ is isomorphic to a structure of the form $R(\alpha), \epsilon\rangle\}$.

It follows from the ineffability that $C$ is stationary, otherwise there is a losed unbounded set $D$ such that $C \cap D=\varnothing$. We can assume $D \subseteq B$. If $, \in D,\left\langle P, \epsilon \cap P^{2}\right\rangle$ is a well-founded structure, elementary equivalent to $R(\beta), \epsilon\rangle$ but it is not isomorphic to a structure of the form $\langle R(\alpha), \epsilon\rangle$. fence by the last lemma there is $x_{P} \in P$ and $X_{P} \subseteq P \cap x_{P}$ such that for no $\in P, t \cap P=X_{P}$.

Define a function $g: P_{k}(R(\beta)) \rightarrow P_{k}(R(\beta))$ by

$$
\begin{array}{ll}
g(P)=X_{P}, & \text { if } P \in D ; \\
g(P)=0, & \text { if } P \notin D .
\end{array}
$$

3y assumption there is $X \subseteq R(\beta)$ such that $E=\{P \mid g(P)=X \cap P\}$ is stationary. Ne can assume $E \subseteq D$. (The intersection of a stationary set and a closed unbounded set is stationary.)

If we define on $E$ a function $P \rightarrow x_{P}$, then by Theorem 3.2 of Jech [2], here is $S \subseteq E, S$ stationary, and $a$ such that for any $P \in S, x_{P}=a . X \subseteq a$ secause $X \cap P \subseteq a$ for every $P \in S$ and $S$, being a stationary set, is unbounded.

$a \in R(\beta)$ and $X \subseteq a$, therefore $X \in R(\beta)$. Pick a $P \in S$ such that $X \in P$ Ind then $X \cap P=X_{P}$ which contradicts the definition of $X_{P}$.

Thus we get that the set $C$ is stationary.

Let $F=\left\{P \mid P \in P_{k}(R(\beta))\right.$, and $k \in P, P \cap k$ is an ordinal $\}$. Clearly $F$ is ilosed and unbounded. Let $P \in C \cap F$. $\left\langle P, \epsilon \cap P^{2}\right\rangle$ is isomorphic to a :tructure of the form $\langle R(\alpha), \epsilon\rangle$. Let $h$ be the isomorphism; $\left\langle P, \epsilon \cap P^{2}\right\rangle$ is an lementary substructure of $\langle R(\beta), \epsilon\rangle$ which proves that $h^{-1}$ is an elementary :mbedding of $\langle R(\alpha), \epsilon\rangle$ into $\langle R(\beta), \epsilon\rangle . P \cap k$ is an ordinal and by induction ve can prove that $h(\alpha)=\alpha$ for $\alpha<P \cap k,|P|<k$, therefore $h(k)=P \cap k<k$; hus $h^{-1}(\alpha)=\alpha$ for $\alpha<P \cap k ; h^{-1}(P \cap k)=k$, which means that $k$ is $\beta$ educible. Q.E.D.

3. Partition property. In $\$ 0$ we mentioned the equivalent definition of neffable cardinal, using some partition property. In this section we show hat this partition property can be generalized and its generalization is zonnected with supercompactness.

DEfinition. $k$ has the $A$ partition property if for any function $A$ on

$$
D_{k}(A)=\left\{\{P, Q\} \mid P, Q \in P_{k}(A), P \subseteq Q\right\}
$$

nto 2 , there is a homogeneous stationary set, that is, a stationary set $B \subseteq P_{k}(A)$ such that $\left|f^{\prime \prime}\left(D_{k}(A) \cap[B]^{2}\right)\right|=1$.

Following Jech [2] we denote the fact that $k$ has the $A$ partition property by $\operatorname{part}^{*}(k, A)$.

THEOREM 2. If $\operatorname{part} *(k, \lambda)$ then $k$ is $\lambda$ ineffable. 
Proof. Let $f$ be a function $f: P_{k}(\lambda) \rightarrow P_{k}(\lambda)$ such that for all $P \in P_{. k}(\lambda)$, $f(P) \subseteq P$. Define $g$ on $D_{k}(\lambda)$ into 2 by $g(\{P, Q\})=0$ iff $P \subseteq Q, f(P) \neq f(Q) \cap P$ and if $\alpha$ is first ordinal in $f(P) \triangle(f(Q) \cap P)$ then $\alpha \in f(Q) \cdot g(\{P, Q\})=1$ otherwise. By part* $(k, \lambda)$ there is a homogeneous stationary set $A$.

Start by assuming the constant value of $g$ on $D_{k}(\lambda) \cap[A]^{2}$ is 0 . We define by induction a set $B \subseteq \lambda$. (Defining a set by induction is defining the relation $\alpha \in B$.) Assume all the relations $\gamma \in B$ for $\gamma<\alpha$ are already defined (that is to say $B \cap \alpha$ is already defined).

We put $\alpha \in B$ iff there exists $P \in A$ such that $\alpha \in f(P)$ and

$$
B \cap \alpha \cap P=f(P) \cap \alpha .
$$

If there exists $P$ satisfying the condition, pick one such $P$ and denote it by $P_{\alpha}$. If there is not $P$ as required, define $\alpha \notin B$.

Claim. $\{P \mid B \cap P=f(P)\}$ is stationary. Otherwise there exists a closed unbounded subset of $P_{k}(\lambda), C$, such that for $P \in C, B \cap P \neq f(P)$.

$C \cap A$ is a stationary set. Define a choice function on this set: $h(P)=$ the first element in $f(P) \triangle(B \cap P)$.

By Theorem 3.2 of Jech, there is a stationary set $D \subseteq C \cap A$ on which $h$ is constant with constant value $\alpha$. For any $P \in D, B \cap P \cap \alpha=f(P) \cap \alpha$ because $\alpha$ is the first element of $B \cap P \triangle f(P)$. Assume $\alpha \notin B$, then $\alpha \in f(P)$ for $P \in D$, but then any member of $D$ satisfies the condition for $P_{\alpha}$ and by definition of $B: \alpha \in B$. Therefore, $\alpha \notin f(P)$ for $P \in D$. Pick $Q \in D$ such that $P_{\alpha} \subseteq Q,\left\{P_{\alpha}, Q\right\} \in D_{k}(\lambda) \cap[A]^{2}$. Hence $g\left(\left\{P_{\alpha}, Q\right\}\right)=0$.

By definition of $P_{\alpha}: P_{\alpha} \cap B \cap \alpha=f\left(P_{\alpha}\right) \cap \alpha$ and $\alpha \in f\left(P_{\alpha}\right)$. But $f(Q) \cap \alpha=$ $B \cap Q \cap \alpha$ which implies (since $\left.P_{\alpha} \subseteq Q\right) f(Q) \cap P_{\alpha} \cap \alpha=B \cap P_{\alpha} \cap \alpha=f\left(P_{\alpha}\right) \cap \alpha$.

Hence, $\alpha$ is the first element of $f(Q) \cap P_{\alpha} \triangle f\left(P_{\alpha}\right)$ but $\alpha \in f\left(P_{\alpha}\right)$ which contradicts $g\left(\left\{P_{\alpha}, Q\right\}\right)=0$.

The other case we deal with is that $g(\{P, Q\})=1$ for $\{P, Q\} \in D_{k}(\lambda) \cap[A]^{2}$. Similarly to the previous case we define $B$ by induction: $\alpha \notin B$ iff there is $P \in A$ such that $\alpha \in P, \alpha \notin f(P)$, and $B \cap \alpha \cap P=f(P) \cap \alpha$. We denote such a $P$ if it exists by $P_{\alpha}$. In any other case, we define $\alpha \in B$. As in the first case we get from the assumption that $\{P \mid B \cap P=f(P)\}$ is not stationary, a stationary set $D \subseteq A$ and $\alpha<\lambda$ such that for $P \in D, \alpha$ is the first element in $f(P) \triangle(B \cap P)$. Hence, for $P \in D, f(P) \cap \alpha=B \cap P \cap \alpha$. Assume $\alpha \in B$, and $\alpha \notin f(P)$ for $P \in D$. Then any $P \in D$ such that $\alpha \in P$ satisfies the condition of $P_{\alpha}$, which implies by definition of $B, \alpha \notin B$. But if $\alpha \notin B, \alpha \in f(P)$; for every $P \in D$ pick $Q \in D$ such that $P_{\alpha} \subseteq Q$. We get that $\alpha$ is the first element in $f\left(P_{\alpha}\right) \triangle(f(Q) \cap P)$ and $\alpha \in f(Q)$ which contradicts $P_{\alpha}, Q \in A$. Q.E.D.

THEOREM 3. $k$ is supercompact iff part $*(k, \lambda)$ for every $\lambda$. 
Proof. If $\operatorname{part}^{*}(k, \lambda)$, then by Theorems 1 and $2, k$ is supercompact. If $k$ is $\lambda$ supercompact then $\operatorname{part}^{*}(k, \lambda)$ which follows from the following theorem by T. K. Menas [5].

THEOREM. If $k$ is supercompact, then there exists a normal ultrafilter $U$ on $P_{k}(\lambda)$ such that for any function $f: D_{k}(\lambda) \rightarrow 2$ there exists a set $A \in U$ and $f$ is constant on $D_{k}(\lambda) \cap[A]^{2}$. Q.E.D.

\section{REFERENCES}

1. G. Fodor, On stationary sets and regressive functions, Acta Sci. Math. (Szeged) 27 (1966), 105-110. MR 34 \#66.

2. T. J. Jech, Some combinatorial problems concerning uncountable cardinals, Ann. Math. Logic 5 (1973), 165-198.

3. R. B. Jensen and K. Kunen, Some combinatorial properties of $L$ and $V$ (mimeographed).

4. M. Magidor, On the role of supercompact and extendible cardinals in logic, Israel J. Math. 10 (1971), 147-157. MR 45 \#4966.

5. T. K. Menas, $A$ partition theorem for $P_{k}(\lambda)$ (mimeographed).

6. W. Reinhardt and R: Solovay, Strong axioms of infinity and elementary embeddings (to appear).

Institute of Mathematics, Hebrew University of Jerusalem, JeRUSALem, IsRael

Current address: Department of Mathematics, University of California, Berkeley, California 94120 